\title{
Investigation of the role of the GABRG2 gene variant in migraine
}

\author{
Timothy Chen ${ }^{1}$, Melanie Murrell ${ }^{1,2}$, Javed Fowdar ${ }^{1}$, Bishakha Roy ${ }^{1}$, Rebecca \\ Grealy ${ }^{1}$, Lyn R. Griffiths ${ }^{1}$ \\ ${ }^{1}$ Griffith Health Institute, Genomics Research Centre, Griffith University, Queensland, \\ Australia. \\ ${ }^{2}$ Australian Prostate Cancer Research Centre, Queensland University of Technology, \\ Brisbane.
}

\section{Correspondence to: Prof Lyn R. Griffiths \\ Director, Griffith Health Institute and Genomics Research Centre School of Medical Science Griffith University Gold Coast Campus \\ Tel: +61755528664 \\ Fax: +61755529081}

Keywords: GABRG2; Gene association; GABA-A receptor; migraine.

\begin{abstract}
Migraine is the most common neurological disorder worldwide affecting about $12 \%$ of the worldwide population. This disorder has been classed into two main types of migraine - with and without aura. While a number of factors can influence the onset of migraine, a major factor is that of genetics. The GABAA gene encodes for the GABAA receptor. Along with other receptors, the GABAA receptor is involved in the mediation of neuronal activities. In this study, a GABRG2 gene (GABAA receptor gamma-2-subunit) SNP (rs211037) was genotyped on a migraine case-control population of 546 (273 affected and an equal number of healthy) individuals. Using specifically designed primers, a high resolution melt (HRM) assay was carried out in the genotyping process. After genotyping, results were compared in the case and control populations. Analysis of results showed no significant differences in the allele frequencies between case and control populations. Similarly no differences were detected for subtypes or for a specific gender of migraine $(\mathrm{p}>0.05)$. Although this gene has been previously found to be involved in febrile seizures and there is some co-morbidity between epilepsy and migraine, we decided to investigate this marker for involvement in migraine. The results did not support a role for the tested GABRG2 variant in migraine.
\end{abstract}

\subsection{Introduction}

Migraine is characterized by recurring attacks of throbbing headaches ranging from mild to severe pain. Migraine affects approximately $12 \%$ of the worldwide population and is 
classed into two general groups of migraine with aura (MA) and migraine without aura (MO). Broken down by gender, migraine affects approximately $6 \%$ of males versus $18 \%$ of females [1]. Generally, the prevalence of migraine increases with age, peaking at approximately 30 to 40 years of age before decreasing to a lower level. Throughout all ages, females have notably higher prevalence in migraine than that of their male counterparts. During attacks, $90 \%$ of migraine sufferers have reported a reduced ability to function normally, with one-third of sufferers requiring bed-rest [1].

Migraines can be influenced by genetic components, and it is thought that specific genes could activate an underlying mechanism causing or aggravating the symptoms of migraine [2]. The gamma-aminobutyric acid (GABA) receptor genes encode for receptors which bind to inhibitory neurotransmitters. Defects in the GABA genes affect the binding actions of the GABA receptor and can lead to hyper- or hypo-excitability. Both actions are thought to have the potential to provoke the symptoms of migraine [3]. As migraine is a complex disease, it can be both multifactorial and polygeneic. The interaction between specific genes could bring about the onset of migraine with genes affecting the pathophysiology [4].

The GABAA receptor is made up of five transmembrane domains - two alpha and beta domains, and one gamma domain. The entry of chloride ions $(\mathrm{Cl}-)$ results in the inhibitory functions of the receptor. This entry is mediated by the binding of GABA neurotransmitter benzodiazepine (BZ) [5]. The gamma-2 subunit of the GABAA receptor is encoded by the GABRG2 gene. This gene is located on chromosome 5q31.1-q33.1 [6] and is the focus of this study. A SNP within exon 5 of the GABRG2 gene (rs211037) has, on numerous occasions been associated with febrile seizures. Due to the symptomatic similarities between some epilepsies and migraine, this SNP was selected as a candidate to investigate for potential associations with migraine [7], [8].

\subsection{Material and Methods}

\subsection{Case samples}

The case-control population consisted of 273 individuals diagnosed with MA or MO and 273 healthy individuals (totalling 546 samples, out of which 245 migraineurs and 247 controls were successfully genotyped). This population was age ( \pm 5 years of age), gender and ethnicity matched (Caucasian population) to the tested case population. All subjects provided a detailed questionnaire and were diagnosed by a clinical neurologist as either MA or MO. The diagnosis of MA or MO was determined using the International Headache Society (HIS) [9]. Genomic DNA was extracted from blood samples from each participant using a method based on a salting out method [10]. All participants provided informed consent and approval was provided by the Griffith University Ethics Committee for research involving human subjects. 
Out of 273 individuals in our migraine case cohort, $214(78 \%)$ had a family history of

migraine and $46(17 \%)$ did not have a family history of migraine. The remaining 13

individuals had not provided us with that information in the questionnaire.

\subsection{Genotyping}

DNA was amplified using the High Resolution Melt method (HRM) utilizing the genomic DNA. Genotyping of the GABRG2 SNP (exon 5/rs211037) was possible by the amplification of this region. Specifically designed primers were used in this study (forward primer sequence: 5'-GAC AAT TGA TGC TGA GTG CC-3', reverse primer sequence: 5'-AGG GGC AGG AGT GTT CAT C-3'). Three possible genotypes were determined: CC, CT and TT. Genotyping results were detected via the Rotor-Gene 6000 Series Software 1.7 (Corbett Research).

Prior to genotyping of the case-control population, the genotypes of positive control or base-line samples were determined. To confirm the amplification of the desired region, the positive control samples were resolved on a $2 \%$ agarose electrophoresis gel at 90 volts for 45 minutes. This yielded an amplicon size of $65 \mathrm{bp}$. Furthermore, the positive control samples were sequenced to verify genotype profile. Prior to sequencing these, samples were purified (QIAQuick PCR purification kit) and underwent the Big-Dye Terminator reaction (BDT) (Applied Biosystems). Upon genotype determination of all positive control samples (seven in total), each of the 3 genotypes were included with the genotyping of the case-control samples as a quality control and base-line comparison.

\subsection{Statistical analysis}

Chi-square analysis $\left(\chi^{2}\right)$ of genotypic and allelic results was undertaken. Prior to analysis the genotype distributions was checked for Hardy-Weinberg equilibrium with no violations to this equilibrium found. Chi-squared statistical analysis was undertaken using the SPSS statistical analysis software (version 16.0.1). Of the 492 samples successfully genotyped, a total of 245 and 247 case and control samples were included in the statistical analysis. The remaining samples were excluded from this statistical analysis due to genotyping difficulties.

\subsection{Results}

This study was designed to find a possible association between rs211037, a marker in the GABRG2 gene and migraine. We had collected some co-morbidity data such as stroke information, blood pressure information, heart disease information etc. and noted that only one out of 175 migraineurs reported having epileptic seizures. 
Upon applying the chi-square analysis, it was found that the case and control population had no significant differences between both the genotype frequency $(p=0.91, \chi 2=0.18)$ and the allele frequency $(p=0.72, \chi 2=0.13)$ (Table 1$)$. Also, no significant differences were found in either the genotype and allele frequencies when comparing the migraine case samples with published frequencies available on the NCBI SNP database. The Hardy-Weinberg equilibrium (HWE) test was applied and the equilibrium was not found to be violated (case: $p=0.31, \chi^{2}=1.05$; control: $p=0.20, \chi^{2}=1.61$ ). The values of p $>0.05$ indicates the validity of the HWE (i.e. no significant difference between the observed and expected groups). The results from this test indicate that there does not seem to be any external factors affecting the genotype or allele frequencies of both populations, thus validating the results (Table 1).

More specific chi-square analyses were also conducted. These tested factors including migraine type (MA or MO), and gender (male or female). When tested separately, both MA and MO did not show any significant differences when compared to the control group (MA: $p=0.88, \chi 2=0.26$; MO: $p=0.85, \chi 2=0.03$ ) (Table 2, Table 3). In addition the genders of male and female were also tested separately with no significant differences found in the genotypes when these groups were compared to the control group (males: $\mathrm{p}$ $=0.52, \chi^{2}=1.29$; females: $\mathrm{p}=0.98, \chi^{2}=0.05$ ) (Table 4, Table 5).

\subsection{Discussion}

Migraine is a debilitating disease affecting approximately $12 \%$ of the population. Specific genes are thought to play a significant role in migraine but as yet all these have not yet been defined. Defects in some $\mathrm{GABA}_{\mathrm{A}}$ receptors have been found to be a potential contributing factor to migraine. These defects may be caused by genetic mutations thus suggesting genetic components of migraine [11].

The GABRG2 gene C588T SNP with an rs value of rs211037 was investigated for its association with migraine. This was accomplished through a case-control approach by the genotyping of 245 individuals with a confirmed diagnosis of MA or MO and 247 healthy individuals. No Hardy-Weinberg violations were found and through a Chi-Square goodness-of-fit test no associations were found between migraine and the genotype and allele frequencies of normal controls.

Changes in the GABA-A gene have been found to potentially alter neuronal excitability. Mutated GABA-A receptors caused by defected genes have been known to cause receptor depolarisation, potentially causing symptoms experienced in migraine [12]. Previous studies on the GABA-A receptor gene have produced evidence suggesting a potential role for this the gene on the pathophysiology of MA [11].

Although no associations were found with the tested variant, this SNP has been previously associated with another neurological condition that results in febrile seizures [7], [8]. The known co-morbidity of migraine with epilepsy prompted an investigation of the variant rs211037, in migraine. Although no association of this SNP was identified the 
gene may still, in combination with other genes, affect migraine and thus studies of other variants in this gene and in other migraine affected populations may be warranted. This study only investigated the association of this SNP to the Caucasian ethnicity. As such, it may be beneficial to study this SNP with other ethnicity populations.

\section{Acknowledgements}

This research was supported by a Queensland state government International Science Linkages grant to investigate potential migraine genes. 


\section{References}

[1] Lipton, R., Bigal, M., Diamond, M., Freitag, F., Reed, M., Stewart, W. Migraine prevalence, disease burden and the need for preventive therapy. NEUROLOGY 2007:68;343-349.

[2] Wessman, M., Terwindt, G., Kaunisto, M., Palotie, A., Ophoff, R. Migraine: a complex genetic disorder. Lancet Neurol 2007:6;521-532.

[3] Fernandez, F., Esposito, T., Lea, R., Colson, N., Ciccodicola, A., Gianfrancesco, F., Griffiths, L. Investigation of Gamma-aminobutyric acid (GABA) A receptors genes and migraine susceptibility. BMC Med Genet 2008:9;109.

[4] Lemos, C., Mendonça, Pereira-Monteiro, J., Barros, J., Sequeiros, J., Alonso, I., Sousa, A. BDNF and CGRP interaction: Implications in migraine susceptibility. Cephalalgia 2010:10.

[5] Jacob, T., Moss, S., Jurd, R. GABA-A receptor trafficking and its role in the dynamic modulation of neuronal inhibition. Nature Reviews 2008:9;331-343.

[6] Lu, Y., Wang, X. Genes associated with idiopathic epilepsies: a current overview. Neurological Research 2009:31;135-143.

[7] Chou, I., Peng, C., Huang, C., Huang, C., Tsai, J., Tsai, F., Tsai, C. Association analysis of $\gamma 2$ subunit of $\gamma$-aminobutyric acid type A receptor polymorphisms with febrile seizures. Paediatric Research 2003:54(1);26-29.

[8] Greenberg, D., Pal, D. The state of the art in the genetic analysis of the epilepsies. Curr Neurol Neurosci Rep. 2007:7(4);320-328.

[9] International Headache Society (IHS) The International Classification of Headache Disorders - 2nd Edition. Cephalgia; 24 (Suppl. 1) 2004: pp. 1-49.

[10] Miller, S., Dykes, D., Polesky, H. A simple salting out procedure for extracting DNA from human nucleated cells. Nucleic Acids Res 1988:16(3);12-15.

[11] Russo, L., Mariotti, P., Sangiorgi, E. Giordano, T., Ricci, I., Lupi, F., Chiera, R., Guzzetta, F., Neri, G., Gurrieri, F. A new susceptibility locus for migraine with aura in 15q11-q13 genomic region containing three GABA-A receptor genes. Am. J. Hum. Genet. 2005:76(2);327-333.

[12] Lüscher, B., Keller, C. Regulation of GABA-A receptor trafficking, channel activity, and functional plasticity of inhibitory synapses. Pharmacol Ther.

2004:102(3);195-221. 